\title{
Matrix and sampling effects on quantification of protein biomarkers of drug-induced liver injury
}

\author{
Viktoria Anselm ${ }^{1}$, Cornelia Sommersdorf ${ }^{1}$, Montserrat Carrasco-Triguero ${ }^{2}$, Paula \\ Katavolos $^{2,3}$, Hannes Planatscher ${ }^{1}$, Andreas Steinhilber ${ }^{1}$, Thomas Joos ${ }^{1,4}$, Oliver Poetz ${ }^{1,4 *}$
}

${ }^{1}$ SIGNATOPE GmbH, Reutlingen, Germany

${ }^{2}$ Genentech, San Francisco, CA USA

${ }^{3}$ Bristol-Meyers Squibb, New Brunswick, NJ USA (at Genentech during the conduct of this study) ${ }^{4}$ NMI Natural and Medical Sciences Institute at the University of Tuebingen, Reutlingen, Germany

*Corresponding Author: Oliver Poetz; Phone: +49 (0)7121 744086-1, Email: poetz@signatope.com, orcid.org/0000-0002-1189-9547

\section{Comments on IA-LC-MS/MS, immunoassay, and enzyme activity data}

In this supporting information, we provide figures and tables for correlation of immunoassay and enzyme assay data in different matrices, analyte information on the investigated peptides and their respective peptides (IA-LCMS/MS method), and method comparison results between IA-LC-MS/MS and immunoassays or enzyme assay. Further analyte results and qualification information is provided in Table S1 (XLSX).

\section{Table of contents:}

- Table S1 (XLSX): Analyte concentration and statistical results from IA-LC-MS/MS, immunoassay, and enzymatic activity assay experiments; calibration curves and quality control samples, MS raw file information.

- $\quad$ Figure S1: Correlation of analyte concentration between different sample matrices (immunoassay and enzyme assay results)

- $\quad$ Table S2: Analyte information for the IA-LC-MS/MS method

- $\quad$ Figure S2: Method comparison results (IA-LC-MS/MS versus immunoassays or enzyme assay) 

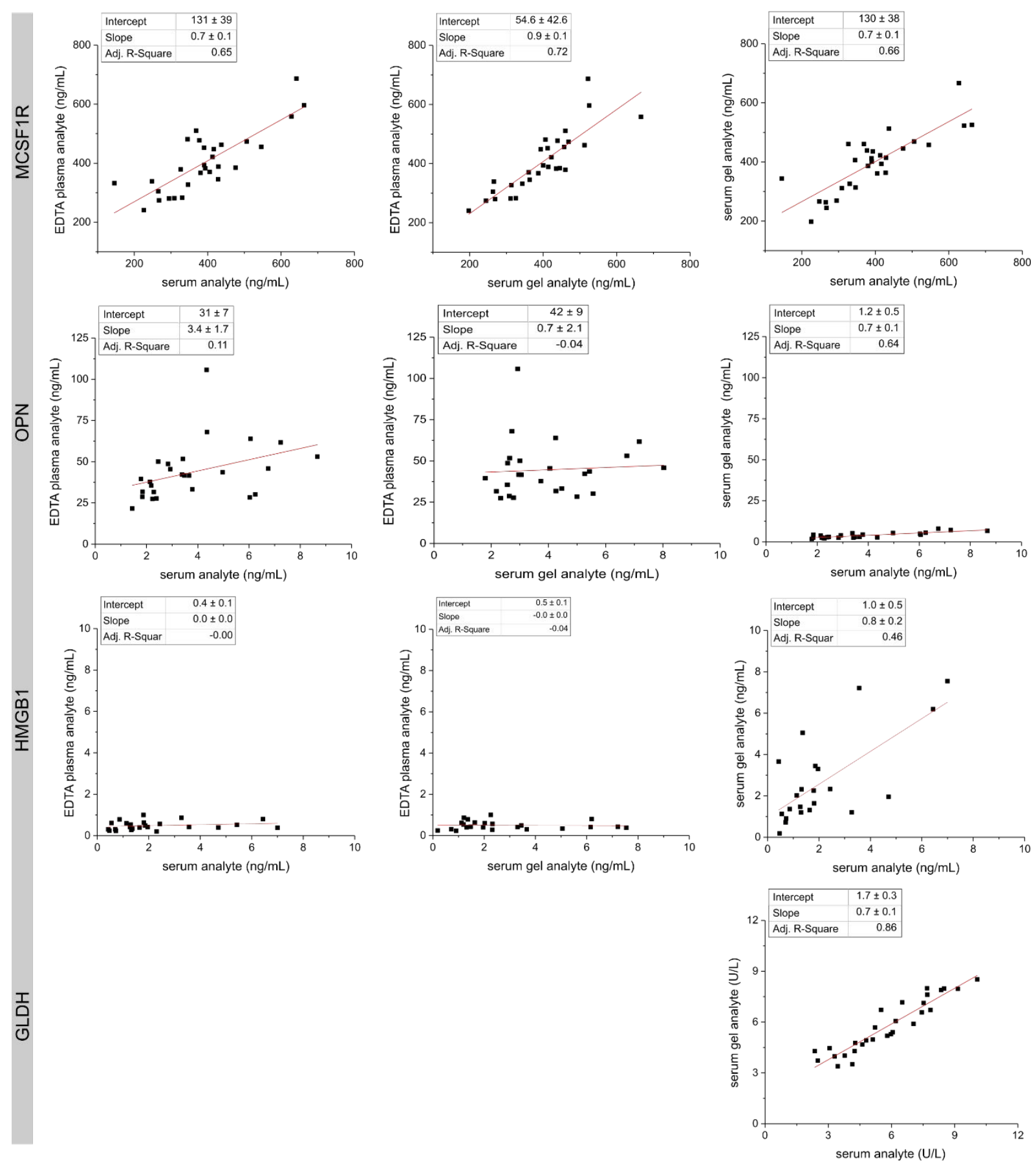

Figure S1. Correlation of analyte concentration between different sample matrices using all time points and matched samples from 10 donors. MCSF1R, OPN, and HMGB1 were measured by sandwich immunoassay, whereas GLDH was measured by enzymatic activity. Outliers were excluded or GLDH (donor 3 ) and only serum and serum gel samples were measured according to the vendor's instruction manual for GLDH. 
Table S2. Analyte information for IA-LC-MS/MS method (isotopically labeled amino acids marked in bold).

\begin{tabular}{llll}
\hline Analyte & MW (Da) & Peptide sequence & Fragment ions \\
UniProt ID & & & \\
\hline MCSF1R & 56981.69 & VIPGPPALTLVPAELVR & $\mathrm{y} 6+, \mathrm{y} 7+, \mathrm{y} 9+, \mathrm{y} 13++, \mathrm{y} 15++$ \\
P07333 & & YPDAVATWLNPDPSQK & $\mathrm{y} 4+, \mathrm{y} 6+, \mathrm{y} 7+, \mathrm{y} 11+$ \\
OPN & 35401.24 & GEHPGLSIGDVAK & \\
P10451 & & & $\mathrm{y} 7+, \mathrm{y} 9+, \mathrm{y} 10+, \mathrm{y} 10++, \mathrm{y} 11++$ \\
HMGB1 & & & \\
P09429 & & HGGTIPVVPTAEFQDR & $\mathrm{y} 8+, \mathrm{y} 9+, \mathrm{y} 11+, \mathrm{y} 12+, \mathrm{y} 14+$ \\
GLDH & & & \\
P00367 & 55973.35 & &
\end{tabular}
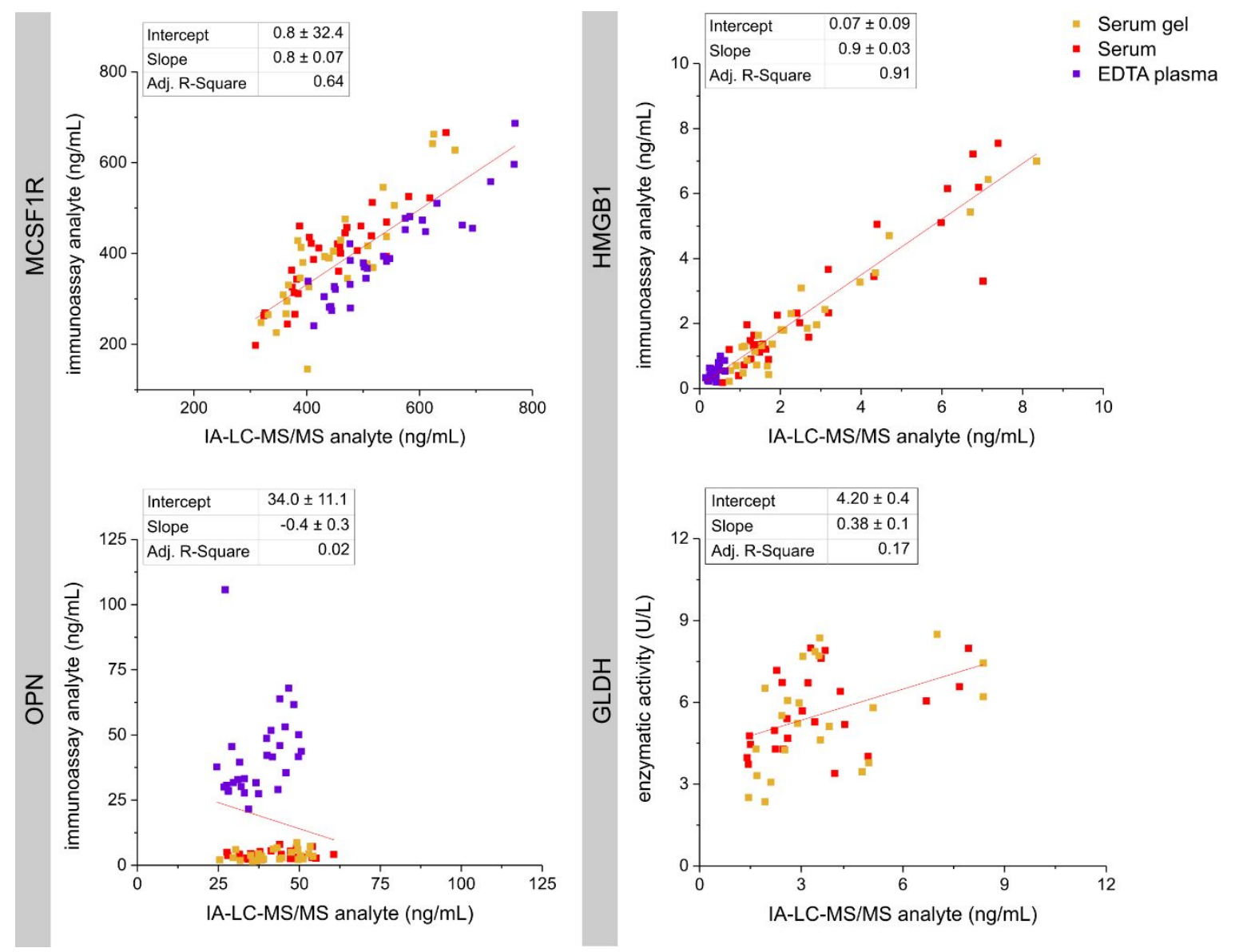

Figure S2. Method comparison. The same samples were analyzed with IA-LC-MS/MS and sandwich immunoassays or by enzymatic activity. Outlier was excluded for GLDH correlation plot (donor 3). For K18 and ccK18, no IA-LC-MS/MS assay was available. 